\title{
ESTRUCTURAS LINGÜÍSTICAS Y PARADIGMAS IDEOLÓGICOS
}

\section{I}

En un trabajo precedente me propuse bosquejar los fundamentos de una tipología textual basada en la teoría lingüística de Louis Hjelmslev. ${ }^{1}$ Me preocupó allí precisar algunos postulados de la glosemática danesa que, a mi modo de ver, proporcionan un firme sustento para la construcción de una teoría literaria que atienda no sólo a los niveles lingüísticos del texto, sino —además-- a los sistemas ideológicos que en él -y por él- se manifiestan. Convendrá revisar sumariamente esos postulados antes de atender a las relaciones que, en cierta clase de procesos, contraen las estructuras lingüísticas y las llamadas "formaciones ideológicas", esto es, las concreciones léxico-discursivas de los valores propuestos por diferentes sistemas de una comunidad cultural.

De acuerdo con Hjelmslev todo proceso o acto de habla tiene uno o más sistemas subyacentes que lo rigen y que nos permiten referir las variantes o fluctuaciones de los diversos procesos a determinadas series de invariantes sistemáticas: los códigos de una lengua. La existencia de tales sistemas o jerarquías de correlatos - a los que Hjelmslev; siguiendo a Saussure, dio el nombre de paradigmáticas - hace posible el desarrollo de los procesos lingǘsticos, es decir, de las sintagmáticas. Una semiótica se definirá, pues, como un proceso sintagmático (o texto) construido con arreglo a las invariantes o códigos de una o más paradigmáticas.

Ahora bien, tanto en las figuras como en los signos de una lengua (para abreviar, las palabras), como en los procesos en que éstos se articulan (frases, oraciones, etc.) pueden distinguirse dos componentes solidarios: el significante y el significado, según la terminología de Saussure; la expresión y el contenido, según la de Hjelmslev. Cada uno de estos funtivos

1 José Pascual Buxó, "Premisas a una semiología del texto literario", en Anuario de Letras, UNAM, 1976; pp. 175-200. 
o términos de la función de signo instituye una forma de la expresión y una forma del contenido, que son arbitrarias respecto de la sustancia - o masa amorfa del sentido- que cada lengua analiza de muy diversa manera; de ahí que tanto la forma de la expresión como la del contenido de un signo lingüistico (ya sea que lo consideremos aisladamente o en articulaciones de mayor extensión $)^{2}$ mantenga una relación arbitraria con el sentido, al cual dan forma en una sustancia de la expresión y una sustancia del contenido. Puede afirmarse, consecuentemente, que

un paradigma de una lengua y otro correspondiente a otra lengua cubren una misma zona de sentido, la cual, aislada de esas lenguas, es un continuum amorfo sin analizar, en el que se establecen los límites por la acción conformadora de las lenguas, ${ }^{3}$

y, a través de ellas, de los sistemas ideológicos sustentados por cada comunidad cultural.

Por otra parte, el análisis semiótico ha de atender al tipo de dependencias que contraigan la expresión y el contenido, ya que ambos funtivos del signo "tienen existencia en virtud de esas dependencias" y por cuanto la totalidad del objeto analizado (el texto) "puede sólo definirse por la suma total de las mismas". (Hjelmslev, Prolegómenos, p. 40). De ahí también que al proceder al análisis textual debamos comenzar por distinguir la forma de la expresión de la forma del contenido y, por lo tanto, la linea de la expresión de la linea del contenido, con el fin de establecer los "dos paradigmas más inclusivos" de la lengua: el plano de la expresión y el plano del contenido. (Cf. Hjelmslev, Prolegómenos, p. 88).

En principio, estos dos planos pueden ser descritos ex-

- Compartimos la opinión de Greimas según la cual "el mismo tipo de relaciones entre semas, registradas en el interior del lexema, puede igualmente existir en el interior de las unidades del discurso más amplias". Cf. A. J. Greimas, Semántica estructural. Gredos, Madrid, 1971; pp. 59 y ss. (Vid. nota 4).

- Louis Hjelmslev, Prolegómenos a una teoría del lenguaje, Gredos, Madrid, 1971; p. 79. 
haustiva y consecuentemente como si estuvieran estructurados de modo análogo, esto es, como si a cada línea del contenido correspondiese una sola línea de expresión $\mathrm{y}$, por lo tanto, como si cada plano de expresión manifestase un solo plano de contenido. Este tipo de dependencias recíprocas (o interdependencias) ocurre efectivamente en las llamadas semióticas denotativas, en las cuales expresión y contenido son de tal modo complementarios que - pongamos por caso- hace del significante araña expresión complementaria del contenido que el Diccionario de Autoridades define como

1)/Araña/ : insecto venenoso de cuerpo pequeño del cual salen de cada lado cuatro zancas o pies largos y delgados.

Pero si de este definición pasamos a

2) $/ \operatorname{Araña} /$ = especie de lámpara fabricada de metal, de madera, de vidrio o cristal, en que se ponen muchas luces,

la complementaridad entre expresión y contenido se ve alterada por cuanto 2) / Araña/ ya no se conecta con el contenido "insecto" sino con el contenido "lámpara". ${ }^{4}$ En casos como éste, diremos que si bien la expresión y el contenido continúan siendo solidarios en el plano sintagmático, no por ello son complementarios en el paradigmático, puesto que cuando seleccionamos el significante araña como expresión del contenido "lámpara", hemos conmutado la invariante sustancial de araña (es decir, "insecto...") por la invariante sustancial "lámpara" y, consecuentemente, habremos desplazado el signo de un paradigma lingüístico a otro.

- Si aceptamos, con Hjelmslev, que "un contenido de signo se equipara a una cadena de contenidos que tengan cierta relaciones mutuas" $y$, además, que "las definiciones [o particiones del significado] con que se traducen las palabras en un diccionario unilingüe son en principio de este tipo" (Prolegómenos, p. 103) nos será posible asumir que -en nuestros ejemplos"insecto" y "lámpara" traducen, respectivamente, dos invariantes de contenido seleccionadas por diferentes sustancias. Tales formas de contenido pueden, por su parte, seleccionar como manifestante una misma forma de expresión (araña). En nuestros ejemplos consideraremos a las cadenas que definen el contenido como la manifestación de invariantes semánticas. 
Tal tipo de dependencias - ya no recíprocas, sino unilaterales- entre expresión y contenido reciben en Hjelmslev el nombre de semióticas connotativas y se distinguen de las semióticas denotativas en que su plano de expresión viene dado por otra semiótica (la conexión complementaria suspendida entre el significante araña y el contenido "insecto") ; por consiguiente, en el contenido de las semióticas connotativas se introducen valores semánticos distintos de los que se actualizan en el contenido de la semiótica denetativa que funciona como su plano de expresión. Dicho de otro modo, al seleccionar /araña/ como expresión del contenido "lámpara", instauramos una parcial homología entre las formalizaciones de dos diferentes zonas de sentido, que se traduce lingüísticamente como la especificación de un miembro o variante del paradigma "insecto" por una variante del paradigma "lámpara", de suerte que el contenido de 2) / Araña/ habrá de actualizar algún aspecto de la forma del contenido de 1) / Araña/, a saber, "la semejanza y figura que tiene [la lámpara] con la araña cuando está extendida", como anota el Diccionario de Autoridades.

Ya se habrá advertido, pues, que la diferencia básica entre una semiótica denotativa y otra connotativa reside en el hecho de que la segunda suspende la relación de complementaridad paradigmática entre un significante y un significado con el fin de que una misma unidad léxica pueda manifestar valores pertenecientes a invariantes paradigmáticas distintas. Las semióticas connotativas constituyen, por lo tanto, un tipo de procesos lingüísticos que se construyen con arreglo a dos paradigmas semánticos, a uno de los cuales corresponden los valores actualizados por la semiótica denotativa, base de la expresión, valores que - en alguna medida - resultan suspendidos por los valores actualizados en el plano del contenido, que especifica al de la expresión.

Las relaciones de especificación que contraen los funtivos del signo (significante y significado) y las partes de una cadena sintagmática (sustantivo y modificadores, núcleo y complementos, etc.) suponen la necesidad de que los hablantes dispongan de un tipo especial de procesos mediante los cuales sea posible traducir a términos de semióticas de- 
notativas ( $A$ : "A"). las relaciones de especificación propias de las semióticas connotativas $\left.\left(A\left[:^{\prime A} \mathrm{~A}\right]\right]: \mathrm{B}^{\prime \prime}\right)$. Estas operaciones, que reciben el nombre de metasemióticas, se distinguen por tener como contenido una semiótica connotativa resuelta conforme a las relaciones de complementaridad entre la expresión y el contenido (o entre el núcleo y sus complementos, etc.) de manera que, volviendo al ejemplo propuesto, la especificación araña $\leftarrow$ "lámpara" se resuelva en la complementaridad lámpara $\leftrightarrow$ "lámpara" (B:"B").

Desde luego, ningún hablante ignora las diferencias existentes entre una lámpara $\leftrightarrow$ "lámpara", una araña $\leftarrow$ "lámpara y una araña $\leftrightarrow$ "araña" siempre y cuando reconozca las equivalencias sémicas que se han establecido entre los respectivos planos del contenido de dichos signos. En otras palabras, que si llamamos comúnmente /lámpara/ a un "vidrio redondo y hondo, más ancho de arriba que de abajo, en el cual se echa aceite y pone una torcida $[\ldots]$ que sirve de mechero" (Diccionario de Autoridades), cuando ese agrupamiento de semas (o rasgos significativos) venga manifestado por medio del significante araña será porque el contenido complementario de éste ("insecto") habrá sido analizado de tal manera que alguno de sus rasgos significativos pueda incluirse en el contenido "lámpara" y, por ende, permita a este último ser especificante del primero.

Ese tipo de conexiones unilaterales entre el plano de la expresión y el plano del contenido del signo - relaciones que habitualmente identificamos como tropos retóricos (metáforas, metonimias, etc.) y que deberemos reconocer como los procedimientos de que se sirven prevalentemente los procesos connotativos- se fundan en características semióticas bien definidas. De cierto, lo que hace posible rescribir los procesos connotativos por medio de operaciones metasemióticas es la peculiar estructuración del plano del contenido, esto es, la agrupación de semas que constituyen el significado de un signo (o semema); agrupación de unidades sig-

- Fundándonos en la terminología de Hjelmslev, llamaremos especificada a la constante de una relación de determinación (esto es, al funtivo de la expresión) y especificante a la variable de dicha relación (es decir, al funtivo del contenido). Cf. Prolegómenos, p. 58. 
nificativas menores que, en términos generales, es homóloga a la combinación de unidades distintivas en el plano de la expresión.

Así, de modo semejante a la combinación de rasgos distintivos en complejos simultáneos (los fonemas) que se enlazan para formar la clase de secuencias que llamamos significantes o planos de expresión del signo, los rasgos significativos se agrupan para formar la clase de complejos simultáneos que llamamos significados o planos del contenido. Y del mismo modo que la conmutación entre los miembros de un paradigma fónico puede dar lugar a un cambio de contenido (dan/van; "dan"/"van"), la conmutación sémica entre miembros de un mismo paradigma semántico trae como resultado un cambio de expresión ("varón" + "adulto" $\leftrightarrow$ hombre; "varón" - "adulto" $\leftrightarrow$ niño). Casos como los que anteceden constituyen las llamadas semióticas denotativas en las cuales se establece una relación de interdependencia entre los funtivos del signo (o de los planos de cada secuencia articulada de signos); pero la lengua conoce también casos en los cuales la forma de la expresión no es complementaria de la forma del contenido que manifiesta, sino que aquella ha sido especificada como significante de ésta. Son las semióticas connotativas en las que se suspende la relación de interdependencia entre los funtivos del signo con el fin de dejar paso a una relación de determinación entre éstos. La determinación implica, por lo tanto, la selección de un miembro ("araña" en el caso ejemplificado) perteneciente a una invariante paradigmática A ("insecto") como expresión de un miembro perteneciente a una invariante paradigmática B ("lámpara"), en cuanto el miembro seleccionado de A ("araña") posea en su contenido algún sema o semas que puedan homologarse con los del contenido del miembro de B.

Como se ve, la sustancia del contenido (o si se prefiere, las entidades extrasemióticas analizadas por un determinado sistema lingüístico) puede ser conformada $a$ ) por medio de conexiones complementarias entre la expresión y el contenido de los miembros que se incluyen como variantes de una misma invariante paradigmática y $b$ ) por medio de co- 
nexiones especificativas entre miembros que se incluyen en invariantes paradigmáticas distintas, especificaciones que se fundan en la suspensión de algún o algunos de los componentes sémicos del miembro seleccionado como significante, y en la consecuente inserción de algunos de sus semas en el contenido del miembro significado.

Charles Sanders Peirce -uno de los fundadores de la ciencia semiótica- observó que cada signo requiere de otro signo o conjunto de signos para ser interpretado, esto es, que el significado de un signo se establece a partir de los signos que pueden traducirlos. ${ }^{\circ}$ En términos lingüísticos, el significado de /araña/, será dado por las traducciones metasemióticas que puedan hacerse por medio de sus interpretantes: /insecto/, /lámpara/, /pez/, etcétera. Pero así como el primer interpretante pone de relieve una relación de complementaridad paradigmática entre la expresión y el contenido (araña $\leftrightarrow$ "araña"), los demás interpretantes nos informan de que ese mismo signo ha sido utilizado como significante de un significado que no es su complementario, sino que le ha sido asignado específicamente $y$, además, que esa particular especificación ha debido fundarse en la conexión araña $\leftrightarrow$ "insecto", es decir, en el desplazamiento de alguno de los interpretantes sémicos que permiten analizar dicha relación de complementaridad.

Por consiguiente, las relaciones unilaterales de determinación entre un signo-significante y un signo-significado dependerán de un desplazamiento paradigmático del primero; vale decir, de la mutación de las zonas de sentido analizadas por un sistema semiótico dado que, cada vez, se pongan de manifiesto. De ahí puede concluirse que la especificación de un signo-significante por un signo-significado presupone la suspensión del agrupamiento sémico del primero en beneficio de uno o algunos de sus rasgos significativos, preci-

- "Un signo, o representamen, es algo que, para alguien, representa algo en algún aspecto o carácter. Se dirige a alguien, esto es, crea en la mente de esa persona un signo equivalente o, tal vez, un signo aún más desarrollado. Este signo creado es lo que llamo interpretante del primer signo". Charles Sanders Peirce, La ciencia de la semiólica, Nueva Visión, Buenos Aires, 1974; p. 22. 
samente de aquellos en que pueda fundarse la especificación del signo-significante, tal como ocurre en nuestro ejemplo araña $\leftarrow$ "lámpara", donde el sema que el Diccionario de Autoridade's interpreta como 'zancas o pies largos y delgados, cada uno de los cuales tiene dos como artejos o nudillos que los dividen en tres partes' ha sido aislado de los demás semas con que se agrupaba en el contenido "araña" y homologado con los del semema "lámpara".

Con todo, no podemos pensar que el tipo de semióticas connotativas a que hemos aludido hasta ahora resulte exactamente equivalente con otra clase de semióticas connotativas que el Diccionario de Autoridades permitiría ejemplificar así:

3)/Araña/ = por translación se llama a la persona que codicia y recoge con solicitud por no buenos modos lo ajeno.

Aquí también la relación entre expresión y contenido es de especificación: el sema 'recoger... por no buenos modos lo ajeno' - de otra manera, 'robar' - ha sido desplazado del paradigma "insecto" al paradigma "hombre" con el fin de producir la semiótica connotativa araña $\leftarrow$ "persona que codicia y recoge..."; pero, en este caso, es evidente que no nos hallamos ante un desplazamiento paradigmático único, es decir, ante la conmutación de una invariante paradigmática por otra, sino que la actividad cazadora de la "araña" ha sido utilizada simultáneamente como significante de valores pertenecientes a un determinado sistema ético-social; en otras palabras, ha servido para actualizar un sistema ideológico cuyos valores desbordan los paradigmas semióticos (lingüísticos), aunque - por otra parte - aquellos hayan de fundarse necesariamente en éstos para alcanzar su propia manifestación.

Para que la conexión araña $\leftarrow$ "hombre" haya podido efectuarse era necesario - como ya advertimos - que alguno de los rasgos significativos de "araña" pudiese ser incluido en el semema "hombre"; este componente sémico homologable es la ocupación del insecto que el Diccionario de Autoridades define como 'fabricar telas y redes con que cazar 
moscas y mosquitos'. En efecto, para que el signo /araña/ pudiese ser especificado como significante de "persona que codicia..." era preciso no sólo conectar dos signos pertenecientes a paradigmas lingüísticos diferentes (el campo semántico de los "insectos" y el de los "hombres") sino, además, evaluar esa actitividad de las arañas desde las mismas perspectivas éticas con que se juzga a los hombres; en otras palabras, implicaba proyectar sobre los valores semióticos de la lengua ciertos valores ideológicos que se ordenan en términos de mandatos y transgresiones en el marco de la vida social. Ello trae como resultado una patente ideologización de los sistemas semióticos, a los que -por otra parte- - las sociedades asignan precisamente la función de servir como vehículos de sus códigos y convenciones de toda índole.

\section{II}

Como se ha visto, la diferencia esencial entre la semiótica connotativa ejemplificada por la relación araña $\leftarrow$ "lámpara" y la que se expresa por medio de la conexión araña $\leftarrow$ "hombre" reside en que la primera supone el desplazamiento de un signo de un paradigma semiótico (lingüístico) a otro, en tanto que la segunda no sólo efectúa esa operación sino que - además - incluye en el contenido de la semiótica connotativa valores pertenecientes a otros sistemas sociales carentes de organización semiótica particular. Por consiguiente, en este segundo tipo de semiótica connotativa no sólo se selecciona un signo perteneciente a un paradigma lingüístico como expresión de otro signo perteneciente a un paradigma lingüístico diverso, sino que se utiliza esta relación especificativa para constelar con el valor que ella manifiesta otros valores pertenecientes a jerarquías ideológicas, que son usualmente verbalizados como/robo/, /codicia/, /hipocresia/, etc. Por constelación entenderemos aquí la correlación de una expresión con un contenido en el cual se hallan combinados miembros pertenecientes a invariantes paradigmáticas distintas ${ }^{7}$, dicho de otro modo, a la clase

"Hjelmslev da el nombre de constelación a "las dependencias de mayor 
de semióticas connotativas en cuyo contenido se manifiestan valores paradigmáticos pertenecientes tanto a sistemas semióticos como a sistemas ideológicos. ${ }^{8}$ Esta parece ser la razón que permite al Diccionario de Autoridades referirse a la actividad cazadora de las arañas con un gesto de repulsión moral no diferente de aquel con que describe la transgresión que un individuo humano puede hacer de ciertos preceptos éticos, religiosos, etc.

Las consideraciones precedentes nos llevan a establecer una radical distinción entre las semióticas connotativas del primer tipo y las del segundo; hemos propuesto dar a estas últimas el nombre de semiologias en virtud de que no se limitan -como las semióticas connotativas propiamente dichas - a manifestar distintos valores paradigmáticos de un mismo sistema semiótico, sino que, además, actualizan en su contenido valores pertenecientes a diferentes sistemas sociales (ideológicos) que son interpretados por medio de los signos propios de un sistema lingüístico dado. En otras palabras, se trata de una clase particular de procesos connotativos que funcionan como metasemióticas no científicas respecto de los valores instaurados por una determinada jerarquía ideológica. ${ }^{9}$

libertad [que las interdependencias y las determinaciones] en las que dos términos son compatibles pero ninguno presupone el otro" (Prolegómenos, p. 42), esto es, a la "función entre dos variables" (id., p. 57). Pero, como hemos observado, la función contraída entre dos variables puede darse a) en el interior de un mismo sistema semiótico, y $b$ ) entre un sistema semiótico y un sistema ideológico que seleccione al primero como interpretante. (Vid. nota 10). Por constelación entenderemos aquí este segundo caso de funciones entre variables de invariantes pertenecientes a sistemas de naturaleza divergente (semióticos e ideológicos).

${ }^{8}$ Las lenguas (o sistemas semióticos) permiten formar un número ilimitado de signos y de combinaciones entre signos, pero las reglas en que se basan los procesos lingüísticos "son independientes de toda escala de valores, sean éticos, lógicos o estéticos; en general, una lengua es independiente de todo propósito específico" (Hjelmslev, Prolegómenos, p. 154). Dichas escalas de valores son -en cambio- postuladas por los sistemas ideologicos y deben ser interpretadas por medio de lenguas. (Vid. nota 12).

- Hjelmsler (Prolegómenos, pp. 168 y ss) distinguió las semióticas cientificas de las semióticas no científicas; las primeras son "operaciones", es decir, descripciones hechas con arreglo al "principio empírico" (autoconsecuencia, exhaustividad y simplicidad), las segundas no son operaciones; consecuentemente, las semióticas denotativas y connotativas no son semió- 
Podemos representar esquemáticamente las relaciones anotadas por medio de los siguientes diagramas:

\section{SEMIOTICA CONNOTATIVA}

Signo-sigrificante signo-significado

\begin{tabular}{|c|c|}
\hline araña & $\begin{array}{c}\text { "támpara" } \\
{[+ \text { "zancas"] }}\end{array}$ \\
\hline 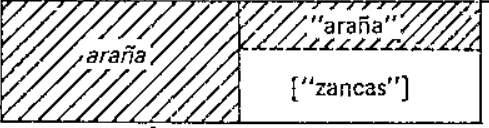 & \\
\hline
\end{tabular}

Semiología

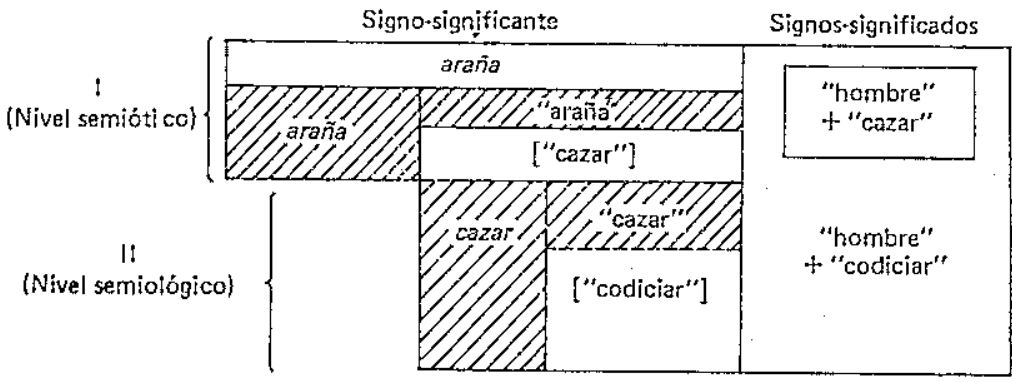

En ambos diagramas, las zonas sombreadas indican las relaciones de complementaridad que han sido suspendidas, y los términos entre corchetes, los sernas desplazados de un paradigma a otro. En el esquema de la semiología, la llave I señala el proceso connotativo básico (nivel semiótico), en tanto que la llave II (nivel semiológico) indica cómo el sema desplazado de I ("cazar") da origen a un segundo proceso connotativo que suspende la especificación entre valores pertenecientes a diferentes paradigmas lingüísticos ("hombre" + 'cazar') con el fin de permitir que el sema

ticas cientlficas, en tanto que las metasemióticas sí lo son. Con todo, existe cierto tipo de procesos metasemióticos no cientificos a los que Hjelmslev dio el nombre de semiologias; podria decirse, pues, que las semiologías son metasemióticas no científicas que tienen por contenido un proceso connotativo a través del cual se manifiestan, simultáneamente, valores de más de un sistema social. 
'cazar' se convierta en signo-significante del contenido "codiciar", esto es, en el interpretante semiótico del valor ideológico que el Diccionario de Autoridades traduce como "procurar con ansia adquirir bienes, riquezas y hacienda por cualesquier medios y modos que uno pueda", vale decir, ilícitamente.

Como puede verse, en el caso de la semiótica connotativa nos hallamos ante un proceso caracterizado por la suspensión de la agrupación sémica del signo-significante en cuanto se considere su relación complementaria con el contenido "araña" y por la predominancia que se concede a uno de los semas actualizados por esa relación, en la medida en que ese sema pueda ser homologado con los del semema "lámpara"; es decir, por la reducción araña < "cuerpo pequeño del cual salen de cada lado cuatro zancas o pies largos y delgados".

En el caso de la semiología estamos también ante un proceso connotativo, por cuanto un sema del signo-significante araña ('cazar [moscas y mosquitos]') se desplaza del contexto paradigmático "insecto" al contexto paradigmático "hombre" para hacer posible la especificación araña $\leftarrow$ "hombre". Pero, como es evidente, esa lectura moralizante de la semiología araña - "hombre" [+ "codiciar"] no sería la única posible, ya que a partir del mismo sema que hemos aislado, el Diccionario de Autoridades puede dar la siguiente interpretación:

4) $/$ Araña $/=$ llámase así metafóricamente al hombre que es muy vividor y provee su casa, recogiendo de todas partes con diligencia y afán,

donde —diacrónicamente- 'vividor' no entraña ninguna condena moral, pues vale por "el que atiende a sus conveniencias y es reservado en los gastos y dispendios". Con todo, tanto en 3) / Araña / como en 4) / Araña/ los semas 'cazar' y 'proveer' han convertido al lexema /araña/ en signo-significante de valores de un sistema ético-social que, por una parte, prohibe a los miembros de la comunidad "codiciar", "robar", etc., y, por otra, los conmina a proporcionar ali- 
mento a quienes dependan de alguno de ellos ("proveer su casa... con diligencia y afán").

En ambos ejemplos aparecen constelados dentro de un mismo proceso sintagmático valores pertenecientes a dos sistemas jerárquicos diferentes (uno semiótico y otro ideológico) ; ahora bien, suponiendo que la especificación araña $\leftarrow$ "hombre" se hubiese producido a partir del desplazamiento de los semas 'cuerpo pequeño del cual salen de cada lado cuatro zancas o pies largos y delgados', tendría como interpretante: "hombre de cuerpo pequeño y de extremidades largas y delgadas" y, por ende, en dicho proceso semiológico se verían simultáneamente actualizados valores pertenecientes tanto a un sistema ético como a otro estético por cuanto 1 -como luego intentaremos precisar- resulta postulable una correlación ideológica entre la malformación física y la imperfección moral.

Abreviando, postularemos que los sistemas que llamamos lenguas, por el hecho de segmentar arbitrariamente la masa informe del sentido, imponen a las realidades extralingüisticas sus particulares estatutos semióticos con el fin de distinguir a las /arañas/ de los demás "insectos" y a las lámparas que son arañas de las que no lo son. Como se ha visto, la homologación de semas constitutivos de signos pertenecientes a diferentes paradigmas es lo que permite seleccionar el significante araña como expresión de contenidos lingüísticos que no se instauran como complementarios suyos; pero, ¿qué es lo que permite a un signo de la lengua convertirse en el significante simultáneo de contenidos jerarquizados por sistemas tanto semióticos como no semióticos? El hecho de que las lenguas naturales no sólo son los únicos sistemas semióticos capaces de traducir a sus propios término todas la demás jerarquías de valores sociales, sino que -ademáspropician el desarrollo de ese tipo de códigos que llamamos ideologías al poner a disposición de éstas el vasto repertorio de semejanzas y oposiciones que las lenguas institucionalizan.

A este propósito decía Emile Benveniste que la relación planteada entre diversos sistemas simbólicos aparece 
determinada ante todo por la acción de un mismo medio cultural, que de una manera o de otra produce y nutre todos los sistemas que le son propios $[\ldots]$ La relación semiótica entre sistemas se enunciará entonces como un nexo entre sistema interpretante y sistema interpretado. Es la que poseemos en gran escala entre los signos de la lengua y los de la sociedad: los signos de la sociedad pueden ser íntegramente interpretados por los de la lengua, no a la inversa. ${ }^{10}$

Pero lo que ahora nos interesa destacar no es tanto la necesidad en que se hallan las ideologías de ser interpretadas por los diferentes sistemas semióticos de una comunidad cultural, sino la manera con que las primeras han permeado el uso lingüístico común al grado de que sus valores específicos suelan aparecérsenos confundidos con los de la lengua e identificados con ellos.

No faltará quien considere que llamar los españoles de los siglos Xvir y xviII araña a una persona codiciosa no pase de ser un hecho lingüístico vulgar que no requiere de mayores averiguaciones para su cabal interpretación. Quienes así piensen no parecerán demasiado sensibles a las múltiples dimensiones que puede desplegar el más inocente de los enunciados $\mathrm{y}$, por lo mismo, no creerán necesario distinguir

10 Émile Benveniste, "Semiología de la lengua", en Problemas de lingüistica general, II, Siglo Veintiuno, México, 1977; pp. 57-58. Benveniste estableció tres tipos de relaciones entre sistemas semióticos: a) de engendramiento que se da entre "dos sistemas de igual naturaleza, el segundo de los cuales está construido a partir del primero" (por ejemplo, el alfabeto normal respecto del alfabeto Braille); b) de homologia, "que establece una relación entre las partes de dos sistemas semióticos" (por ejemplo, entre la arquitectura gótica y el pensamiento escolástico) y c) de interpretancia entre un sistema interpretado y un sistema interpretante; "desde el punto de vista de la lengua, es la relación fundamental, la que reparte los sistemas que se articulan, porque manifiestan su propia semiótica, y sistemas que son articulados y cuya semiótica no aparece sino a través de la reja de otro modo de expresión". (Id., pp. 64-65). Esta última relación es la que permite explicar el hecho de que los sistemas ideológicos hayan de ser manifestados por intermedio de "lenguas" que modelan semióticamente a los restantes sistemas sociales y de que, a su vez, las lenguas se vean semánticamente remodeladas por los sistemas sociales, de manera que la sustancia de contenido de una función de signo no sea ya una mera porción del "continuum amorfo del sentido", sino -a nuestro parecer- una sustancia conformada previamente por algún sistema ideológico. 
uno o más sistemas ideológicos verbalizados en expresiones : como algunas de las que acabamos de analizar, ya que para tales persona la lengua representará siempre una sólo mánera de nombrar y asumir la realidad del mundo, precisamente la única que le consiente su propia conciencia ideológica. Con todo, no podrá dejarse de reconocer que detrás del signo /araña/ empleado como expresión del contenido "hombre codicioso" hay todo un proceso de ideologización de la actividad de las arañas e, inclusive, de antropomorfización moral de los arácnidos, que no sólo se ofrecen como símbolo de cierta clase de individuos sino que se brindan íntegramente como metáfora viva de la "codicia", la "hipocresía", la "poltronería viciosa" y de otras muchas cosas más.

De modo, pues, que si el signo /araña/ convierte un poco en "insecto yenenoso" al hombre a quien se le aplique, de igual manera la especie de los arácnidos se volverá semiológicamente humana cuando se le haga símbolo de un comportamiento moral sancionado por la cornunidad. (Díganlo $\longrightarrow$ si no- tantas fábulas doctrinales).

\section{III}

Volviendo ahora sobre algunas de las cuestiones planteadas, resulta paradójico observar que esa clase de textos que hemos llamado semióticas connotativas parecieran propiciar una vuelta al caos de aquel orden provisorio al cual las semióticas denotativas se empeñaban en reducir la amorfa sustancia del pensamiento. Quiere decirse, pues, que aun cuando tengamos a la vista una lámpara real y concreta, por el hecho de nombrarla araña algo de las arañas se integrará con ella $o$, en otras palabras, que esos objetos que la lengua es capaz de incluir en paradigmas léxicos netamente delimitados, tienden a ser recubiertos e identificados con otros en nuestra conciencia lingüística, que podría seguir manteniéndolos separados y distintos.

En toda semiótica connotativa puede vislumbrarse, como esfúmada en segundo plano, una "imagen conceptual" que altera las referencias del signo, de modo tal que en las se- 
mióticas connotativas queda un significado latente - pero no realmente oculto-debajo del contenido que el enunciado hace emerger hasta el primer plano de la significación.

Es preciso reconocer, sin embago, que en la práctica linz güística estos contenidos de segundo plano se ven generalmente rechazados en beneficio de la inequivocidad del significado prevalente (o complementario), de suerte que el orden semiótico que le ha sido impuesto al mundo no résulte definitivamente transtornado. Pero cuando pasamos de las semióticas connotativas simples a las semiologías, resultan ser esas imágenes neblinosas del transfondo las que emergen a la superficie, provocando así el fenómeno conocido como polisemia o ambigüedad referencial.

Es evidente que, por razones de economía, la lengua puede expresar por medio de un mismo significante muy diversos contenidos; pero esa disponibilidad de los signos para servir de expresión a diferentes valores paradigmáticos no implica que el emitente o el destinatario de un mensaje tengan que constreñir la significación del mismo a una sola serie de valores semióticos con prescindencia de otros valores sociales que ese mismo mensaje haya querido poner de manifiesto. Parece usual que las parejas de oposiciones actualizadas en un determinado mensaje mantengan un mismo tipo de relaciones sistemáticas (ya sean de complementaridad, ya de especificación); pero tanto en la lengua coloquial como -en mayor medida- en la poética, un mismo signo puede insertarse alternativa o simultáneamente, no sólo en más de un paradigma lingüístico, sino en uno ó más sistemas ideológicos que tengan por interpretante un mismo proceso textual. Es el tipo de relaciones que designamos como constelación y que ahora podríamos précisar diciendo que son aquellas que dan lugar a una clase de procesos connotativos que funcionan como interṕretantes de valores instaurados por sistemas sociales carentes de signos particulares o exclusivos.

Así por ejemplo, en la letrilla de Quevedó que dice ${ }^{-4}$. 
y eras araña que andabas

tras la pobre mosca mía,

el signo /araña/ del tercer verso aparece especificado como signo-significante de la /codicia/humana del segundo $y$, además, como significante de "araña" en cuanto se opone al contenido complementario de /mosca/. Por su parte, el significante mosca es expresión del contenido "insecto" (que lo instala en el mismo paradigma léxico al que pertenece la "araña"), pero en cuanto se articula con su especificador muerta es signo-significante de "fingimiento" o "hipocresía" y, en cuanto se articula con los modificadores pobre y mia, es signo-significante del contenido "dinero", como hace constar el Diccionario de Autoridades.

De este modo, las alternativas relaciones de complementaridad y especificación contraidas por /araña/ y por / mosca/ permite la utilización de dichos signos como interpretantes simultáneos (constelados) de diferentes correlaciones sistemáticas que —atendiendo al nivel ideológico-.. podrían ser nuevamente verbalizadas por medio de los siguientes pares de oposiciones o semejanzas: fingir ("hablabas") /hacer ("andabas"); codicia ("araña") / hipocresia ("mosca muerta"); codicia ("araña") dinero ("mosca").

Obviamente, el texto de Quevedo va mucho más allá de estas designaciones cruzadas que, por lo demás, formaban parte de un juego de connotaciones ordinario en el habla coloquial de su tiempo. Pero conviene señalar que la locución mosca muerta —definida por el Diccionario de Autoridades como "apodo que se aplica al que parece de ánimo u genio apagado, pero que no pierde la ocasión de su provecho"- - designa aquí una forma disimulada de la codicia femenina $y$, en última instancia, una plaga social, como confirma el propio Quevedo en aquel soneto que "Reprende en la araña a las doncellas, y en su tela la debilidad de las leyes":

Si en no salir jamás de un agujero y en estar siempre hilando te imitaran las doncellas, joh araña,! se casaran con más ajuar y más doncel dinero. 
Imitan tu veneno lo primero, luego tras nuestra mosca se disparan; por eso, si contigo se comparan, más tu ponzoña que sus galas quiero.

Ya hemos señalado que cuando se emplea el signo /araña/ como significante de "individuo codicioso", la función semiótica que se establece entre el plano de expresión y el plano de contenido pareciera instaurar, no sólo una correlación entre diferentes formas del contenido, sino -además - entre sus respectivas sustancias, hecho que permitiría a la expresión verbal usurpar la entidad que corresponde a sus referentes extralingüísticos. Se trata, desde luego, de un efecto ilusorio provocado por la presunta identidad entre los signos y las cosas designadas; pero esta ilusión se vuelve realidad semiótica cuando la sustancia del contenido de una forma lingüística ya no sea una zona del sentido conformada por la lengua, sino por otra forma de contenido, esto es, cuando las palabras designen zonas de las sustancia social o psíquica previamente formadas por otros sistemas de valores de la comunidad cultural; dicho aún de otro modo, cuando los referentes de un proceso semiótico sean sustancias conformadas por otros sistemas sociales (éticos, religiosos, políticos, estéticos, etcétera), que rigen el comportamiento colectivo y que, por lo tanto, obligan a interpretar toda actividad humana no sólo con arreglo a las normas establecidas por esas paradigmáticas ideológicas, sino a través de los signos semióticos que sean susceptibles de manifestarlas. ${ }^{11}$

Así, cuando Quevedo se propuso expresar poéticamente un universo de valores morales degradados por causa de sus reiteradas si bien encubiertas transgresiones, tuvo que partir

${ }^{11}$ Es evidente que el factor decisivo para la existencia de un proceso es la existencia de un sistema que lo rija; según Hjelmslev, "es inimaginable un proceso - porque sería inexplicable en un sentido absoluto e irrevocable- sin un sistema tras el mismo" (Prolegómenos, p. 62). Pero aun siendo imaginable la existencia de un sistema "virtual" sin que contemos con ningún texto construido a partir de él (cf. id., p. 63), la existencia de un sistema "real" sólo es comprobable a partir de la existencia de sus procesos correspondientes. Consecuentemente, la existencia de sistemas ideológicos "reales" es únicamente deducible de los textos que manifiestan sus jerarquías paradigmáticas. 
necesariamente de los signos lingüísticos que permiten establecer las parejas opositivas del sistema moral en cuestión $y$, consecuentemente, hubo de interpretar semióticamente parejas de valores tales como "humanidad"/ "animalidad", "racionalidad" / "irracionalidad", "espiritualidad"/"instintividad", etcéterá o más concretamente, por medio de lexemas - como /araña/, por ejemplo- algunos de cuyos interpretantes sémicos manifiestan ciertos valores de un determinado sistema ideológico ("codicia", "hipocresía", etcétera). De modo, pues, que la forma semiótica de ese universo de valores degradados había de sustentarse en una permanente alusión a aquellos textos en que previamente se hubiese expresado el estado de acuerdo o equilibrio entre los preceptos morales (los paradigmas ideológicos) y los comportamientos humanos (la actualización sintagmática de los valores paradigmáticos, si puede decirse así). ${ }^{12}$ La observancia de los mandatos morales pudo llevar al hombre al perfeccionamiento de su alma - - la parte que posee en concomitancia con la divinidad, de la que emanan los preceptos-, pero su contumaz desobediencia no podría sino conducirlo a la pérdida progresiva de su condición humana $y$, por ende, a su creciente animalización, la cual —pese a considerarse como

1s Para Hjelmslev ("La estratificación del lenguaje", en Ensayos lingüisticos, Gredos, Madrid, 1972; pp. 47 y ss) la sustancia puede estar semióticamente formada o semióticamente no formada. El primer caso explica que "una misma forma del contenido pueda ser expresada por varias formas de expresión"; el segundo implica que una misma sustancia comporte varios aspectos o niveles (el físico, el sociobiológico y el de las apreciaciones sociales). Por to que toca a la sustancia del contenido, un examen provisional -decía Hjelmslev- "invita a concluir que en el interior de esta sustancia el nivel primario, inmediato, en cuanto único directamente pertinente desde el punto de vista lingüístico y antropológico, es el nivel de apreciación social" (id., p. 69); esto es, el nivel de la sustancia semiológica, ideológicamente formada, que selecciona como manifestante una sustancia semioticamente formada. De hecho, y volviendo al texto citado de Hjelmslev, "la sustancia sémiótica inmediata, que evidentemente selecciona la forma que manifiesta, es seleccionada a su vez por los otros niveles"; y aunque el nivel de apreciación social (o, en nuestros términos, la sustancia semiológicamente formada) "constituya un objeto mal estudiado", su fundamentación teórica habrá de atender al cuerpo de doctrinas $y$ de opiniones adoptadas por la tradición y el uso social, vale decir, por los sistemas ideológicos. 
un fenómeno metafísico- bien puede proyectarse metonímicamente sobre su ser corporal.

Obviamente, nada de esto puede ocurrir fuera de un medio cultural específico ni de la conciencia que sus miembros tengan de los sistemas semióticos que permiten expresar esas peculiares correlaciones de valores semióticos e ideológicos. Se trata, por lo tanto, de realidades eminentemente culturales que -ni más ni menos que los otros géneros de realidades - han de ser necesariamente expresadas e interpretadas por medios semióticos. De ahí que los procesos verbales que llamamos semiologías (y más concretamente las semiologías que son textos poéticos) constituyan los lugares privilegiados del encuentro y la fusión de los signos de una lengua (que permite organizar cualquier sustancia por medio de paradigmas abiertos cuyos miembros son fácilmente permutables) con los valores de los sistemas ideológicos de una comunidad histórica, que organizan determinadas parcelas de la sustancia psíquica y social con arreglo a paradigmas cerrados; es decir, según un conjunto fijo de correlaciones que, sin embargo, resultan expresables en un número indefinido de signos semióticos.

- José Pascual Buxó

Seminario de Poética.

Instituto de Investigaciones Filológicas. 